\title{
Dry Eye Disease: Impact on Quality of Life and Vision
}

\author{
Miki Uchino • Debra A. Schaumberg
}

Published online: 15 March 2013

(c) Springer Science + Business Media New York 2013

\begin{abstract}
Dry eye disease (DED) is a growing public health concern causing ocular discomfort, fatigue, and visual disturbance that interferes with quality of life (QoL), including aspects of physical, social, and psychological functioning, daily activities, and workplace productivity. This article assesses the current understanding of the impact of DED on QoL and vision. The full impact of DED on a patient's QoL is not easily quantifiable, but several methods and techniques have been evaluated to measure the decreased quality of vision from DED, and a number of questionnaires have been developed to quantify the impact of DED on various aspects of patient QoL. We summarize available evidence on the impact of DED from a review of published literature.
\end{abstract}

M. Uchino

Department of Ophthalmology, Keio University School

of Medicine, Tokyo, Japan

D. A. Schaumberg $(\square)$

Division of Preventive Medicine, Brigham and Women's

Hospital, Harvard Medical School, 900 Commonwealth

Avenue East, Boston, MA 02215, USA

e-mail: dschaumberg@ rics.bwh.harvard.edu

D. A. Schaumberg

Department of Ophthalmology \& Visual Sciences,

University of Utah, Salt Lake City, UT, USA

D. A. Schaumberg

Department of Epidemiology, Harvard School of Public Health,

Boston, MA, USA

D. A. Schaumberg

Department of Ophthalmology, Schepens Eye Research Institute, Harvard Medical School, Boston, MA, USA
Keywords Dry eye disease - Quality of life - Quality of vision · Functional visual acuity - Questionnaire

\section{Introduction}

According to the International Dry Eye WorkShop in 2007, "dry eye is a multifactorial disease of the tears and ocular surface that results in symptoms of discomfort, visual disturbance, and tear film instability with potential damage to the ocular surface" $\left[1^{\bullet}\right]$. Dry eye disease (DED) is one of the most prevalent ocular surface diseases in the world. The prevalence of DED has been reported to occur in the range from approximately $4.4 \%$ to as high as $50 \%[2-7$, 8•, 9] among middle-aged and older people throughout the world. In the USA, estimates from the largest studies suggest that DED affects around five million people aged 50 years or older [8•]. Symptoms of DED are a common motivation for patients to seek eye care, and have emerged as crucial outcome measures for clinicians as well as in studies assessing the impact of DED treatments. Accordingly, measurement of the impact of DED on patients' daily lives is now recognized as a critical aspect of disease characterization.

Researchers in the field have worked to develop increasingly robust ways of measuring patient-reported outcomes in DED. With the improvement and expansion of available techniques to evaluate the impact of DED on people's lives, there is a growing body of evidence detailing the depth and breadth of the impact of DED on quality of life (QoL) and vision. A major impact of DED, as included in its definition, is its effect on visual function. People with DED often report visual disturbances such as blurred or foggy vision, fluctuating vision, and glare; often in spite of normal visual acuity using standard testing 
techniques. The resulting reductions in visual function can be measured by questionnaires [10-12], contrast sensitivity tests $[13,14]$, functional visual acuity (FVA) tests [15-21], and measurement of higher-order optical aberrations [2225]. DED also impacts other aspects in the everyday QoL of patients, including physical, social, and psychological functioning and workplace productivity. The physical impact of DED seems most closely related to the concept of DED as a type of chronic pain syndrome, which results in chronic symptoms of ocular surface discomfort with subsequent effects on a number of aspects of QoL.

\section{QoL as Assessed by Questionnaires Developed Specifically for DED}

In recent years, a number of patient-reported outcomes questionnaires have been developed to assess patients' experience in DED [10, 12, 26]. These questionnaires include those directed at measuring ocular surface comfort symptoms, e.g., the Symptom Assessment in Dry Eye (SANDE) questionnaire, which is based on a visual analogue scale approach, and the Ocular Comfort Index (OCI) questionnaire, which was developed using the technique of Rasch analysis, as well as those aimed at measuring both ocular surface comfort symptoms and the impact of DED on other aspects of a patient's QoL. We describe a few such questionnaires below.

For example, the Ocular Surface Disease Index (OSDI) [10] consists of 12 questions and has been frequently used to measure the severity of DED, including in the setting of clinical trials. The OSDI contains three subscales: ocular discomfort symptoms, vision-related function, and environmental triggers, which are all queried by three or more questions which direct patients to their experience over the past week. More specifically, the OSDI includes three items related to ocular discomfort (feeling sensitive to light, gritty, and painful or sore eyes) and six questions related to visual disturbance (blurred vision or poor vision) or visual function (problems reading, driving at night, working with a computer, or watching TV), and three questions related to possible symptom triggers (windy conditions, low humidity, or areas that are air-conditioned).

The OSDI was shown to validly distinguish among patients with no DED and patients with mild, moderate, and severe DED [10]. It has since been used in a number of research articles and randomized trials [27-29], and data from the OSDI demonstrate the higher level of ocular surface symptoms in patients with DED. For example, a comparative study including 87 patients with DED showed that the dry eye patient group had higher (worse) OSDI composite and subscale scores of ocular symptoms, visionrelated function, and environmental triggers (all $P<0.001$ ) than a group of 71 patients without DED [27]. A study with 40 participants with clinical evidence of DED showed that the OSDI total score was significantly greater in patients with DED than in normal subjects [30].

The Dry Eye Questionnaire (DEQ) [26] includes 21 items developed to evaluate the prevalence, frequency, diurnal severity, and intrusiveness of dry eye symptoms for use in epidemiologic and clinical studies. The questions inquire about the frequency of ocular discomfort, dryness, visual changes, soreness and irritation, grittiness and scratchiness, burning and stinging, foreign body sensation, light sensitivity, and itching. It also includes questions on age, gender, daily activities, computer use, use of systemic and ocular medications, allergies, self-assessment, and previous diagnosis of dry eye. There are four questions in the DEQ related to visual disturbance, such as frequency of visual changes, how noticeable the changes are during the morning or before bed, and how much the visual fluctuation bothers the patients. A study with 100 patients using the DEQ found symptoms of ocular irritation were frequent and intense among patients with Sjögren syndrome and keratoconjunctivitis sicca compared with controls. These symptoms often increased in intensity over the day, suggesting that open-eye conditions affect the progression of symptoms [26].

Another questionnaire used for epidemiologic and clinical studies, the Impact of Dry Eye on Everyday Life (IDEEL) [12],consists of 57 questions comprising three modules: dry eye symptom bother; impact on daily life, including daily activities, emotional impact, impact on work; and treatment satisfaction (both effectiveness and treatment-related bother/inconvenience). The questions items to visual disturbance are two items on how much the person is bothered by "blurry vision" or "sensitivity to light, glare, and/or wind." Authors have observed statistically significant differences in responses to the IDEEL questionnaire across differing levels of DED severity [12]. One study with 74 subjects found IDEEL-symptom bother significantly discriminated dry eye severity, and after the treatment of DED the IDEEL-symptom bother dropped among subjects with improvement [31].

In the clinical setting, in addition to symptoms of ocular surface pain and discomfort, physicians often encounter patient complaints of blurred vision even though the patient's best-corrected visual acuity is normal when measured with a Snellen or other standard visual acuity chart. Consistent with this, studies of patients with DED using patient-reported outcome instruments have consistently identified problems with performance of daily activities that require sustained visual attention. For example, a study using the DEQ found that $10 \%$ of patients with non-Sjögren syndrome DED and $30 \%$ of patients with Sjögren syndrome DED [32] complained about impaired vision. Others have reported that between 
42 and $80 \%$ of patients with primary Sjögren syndrome experienced "disturbed vision" [33, 34]. In addition, a study from Japan showed that blurred vision was reported in $22 \%$ of DED patients [35].

In one of the first studies of its kind, data from a subset of participants from the Women's Health Study [8•] and the Physicians' Health Study [36] demonstrated that people with DED are significantly more likely than people without DED to report problems with reading [odds ratio (OR) $3.64,95 \%$ confidence interval (CI) 2.45-5.40], performing professional work (OR 3.49, $95 \%$ CI 1.72-7.09), computer use (OR 3.37, $95 \%$ CI 2.11-5.38), watching television (OR 2.84, $95 \%$ CI 1.05-7.74), daytime driving (OR 2.80, $95 \%$ CI 1.58-4.96), and nighttime driving (OR 2.20, $95 \%$ CI 1.48-3.28) [37•] (Fig. 1). A later study from Singapore with 3,239 individuals aged 40 years or older showed that patients with symptomatic dry eye reported significantly more difficulty in performing vision-related daily activities, independent of their visual acuity. The specific activities that were affected by symptomatic dry eye were navigating stairs, reading road signs, reading newspapers, cooking, recognizing friends, watching television, and driving at night [38].

\section{Use of Utility Assessments in DED}

Utility assessment is a formal method for quantifying and understanding the relative impact of a given health state or disease on patients. One of the advantages of this technique is that utility scores that are anchored at perfect health (utility 1) and death (utility 0) can be compared across various health outcomes using a time trade-off (TTO) method [39]. Two studies used the technique of utility assessment to estimate the impact of DED on QoL, finding that for patients with severe DED, the impact of their disease on their lives was similar to the impact of moderate to severe angina [40, 41]. Schiffman et al. [41] found that for the severest DED cases (requiring tarsorrhaphy), the impact was worse than that reported for disabling hip fracture. Buchholz et al. [40] from the UK showed a similar result; 44 patients with moderate to severe dry eye were surveyed via interactive utility assessment software. Utility values were measured by TTO and standard gamble methods. With the TTO method, the mean score for asymptomatic dry eye $(0.68)$ was similar to that for "some physical and role limitations with occasional pain" and severe DED requiring surgery scored 0.56 , similar to hospital dialysis (0.56-0.59) and severe angina (0.5). Utilities described for scenarios of dry eye severity levels were slightly higher (less severe impact) for patients selfreported as having mild to moderate DED versus those self-reported as having severe DED.

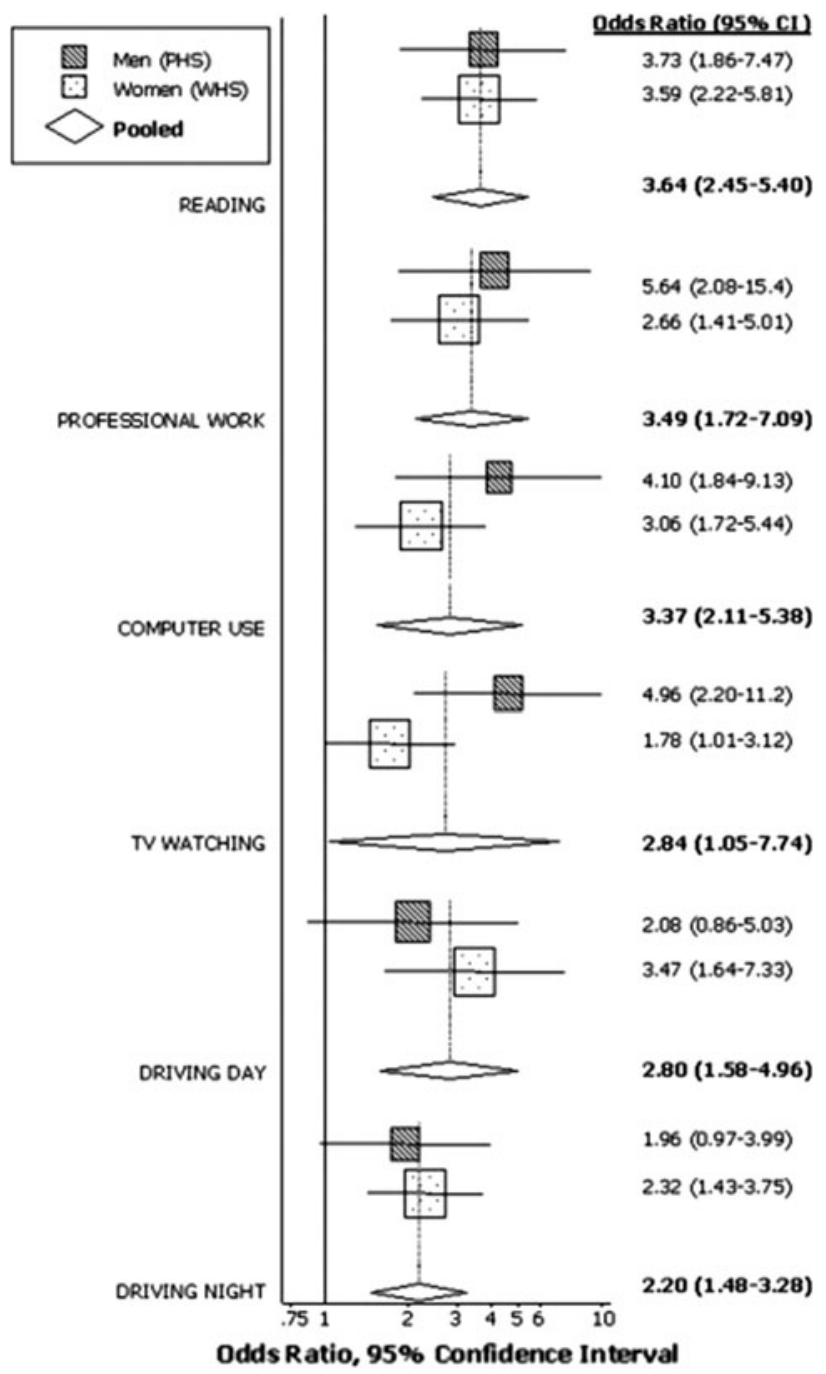

Fig. 1 Impact of dry eye disease on vision-related quality of life. $C I$ confidence interval, $P H S$ physicians' health study. (From [37•] with permission)

\section{Use of Generic Health or Generic Eye Health Questionnaires in DED}

The 36-Item Short Form Health Survey (SF-36) [42] questionnaire is a widely accepted generic metric for assessment of health-related QoL that has been applied in the setting of DED. The SF-36 is a multipurpose, short-form health survey with 36 questions. It yields an eight-scale profile of functional health, including physical functioning, role limitation because of physical disability, bodily pain, general health, vitality, social functioning, emotional limitation because of emotional disability, and mental health. These measures are summarized by a physical component summary score and mental component summary score. Patient responses to the SF-36 showed that DED has a measurable negative impact on such general health assessments. Indeed, in one study, even patients with mild DED had consistently lower 
physical role functioning and bodily pain scores than normal patients, whereas patients with moderately severe disease experienced not only these losses but also measurable reductions in reported vitality and poorer general health. The group with severe DED scored lower than the normal group across all SF-36 domains except emotional role functioning and mental health [43]. A link of DED with emotional and mental health has also been demonstrated with other methods, for example, an association between the use of antidepressant medication and risk of DED was observed in the Physicians' Health Study [36], and this finding is also consistent by data from at least two other epidemiologic studies $[44,45]$.

Generic visual function questionnaires such as the National Eye Institute's Visual Function Questionnaire 25 (NEI VFQ-25) have also been used in many studies of DED [27, 46-48]. The NEI VFQ-25 is a 25-item questionnaire with five nonvisual domains - general health, mental health, dependency, social function, and role limitations-and seven visual domains-general vision, distance vision, peripheral vision, driving, near vision, color vision, and ocular pain. It has been shown to be a useful tool for grouplevel comparisons of vision-targeted, health-related $\mathrm{QoL}$ in clinical research for multiple eye conditions. Of specific interest for DED, the questionnaire contains items on visionrelated QoL as well as two ocular pain subscale questions. Nichols et al. [48] used the NEI VFQ-25 to study visionrelated QoL among predominantly mild to moderate DED patients, particularly in relation to reported ocular pain. In these patients, the pain and discomfort subscale of this instrument had the lowest score of all the subscales $(83.8 \%)$. Further, a population-based cross-sectional study from China with 229 patients shows that NEI VFQ-25 scores were significantly lower in subjects with $\operatorname{DED}(P=0.003)$. Moreover, this study found the subscale scores of ocular pain and mental health were significantly lower in those with either DED or dry eye symptoms only (both $P<0.001$ ) [47]. Another study showed that the DED group had worse NEI VFQ-25 scores for the subscales of general health, general vision, ocular pain, short-distance-vision activities, long-distance-vision activities, vision-related social function, vision-related mental health, vision-related role difficulties, vision-related dependency, and driving [27]. The NEI VFQ-25 subscale scores were generally low, with significantly lower scores for general health, general vision, and ocular pain [27]. The low NEI VFQ-25 scores reported by patients with DED in that study correspond well to those reported by Nichols et al. [48] and Vitale et al. [49].

It also deserves mention that patients with topically treated glaucoma present with DED more often than a similar control group, and the presence of DED negatively influences QoL in this group of patients over and above the presence of glaucoma itself [50].
The prevalence and the risk of DED appears to be higher in glaucoma patients [51], and an observational crosssectional study with 61 patients who were treated with glaucoma medications showed that QoL measured by the NEI VFQ-25 was significantly reduced among glaucoma patients who also developed DED [50].

\section{Contrast Sensitivity Measurement}

Contrast sensitivity tests measure the ability of the eye to discern between aspects of different brightness in an image; patients with poor contrast sensitivity may have difficulty identifying details in patterns or distinguishing facial features. In fact, the visibility of an object is often limited more by its contrast than by its size, which can be demonstrated on certain contrast sensitivity charts. Insofar as contrast sensitivity tests measure the ability to distinguish objects from their background, they provide additional information about the visual system than can be obtained by visual acuity measurement alone. With the use of Vistech chart, which presents sine-wave gratings at set contrast levels, Rolando et al. [52] found a significant decrease in contrast sensitivity in DED patients with punctuate epithelial keratopathy compared with patients without it. Using a chart-based system that presented sinewave gratings of fixed contrast, Huang et al. [13] reported that contrast sensitivity in DED patients decreased regardless of the presence of punctuate epithelial keratopathy. Ridder et al. [53] employed computer-generated sinewave gratings that were briefly presented (16-ms duration), and demonstrated that DED patients exhibit a decrease in contrast sensitivity when the tear film breaks up. These measurable changes in contrast sensitivity are indicative of some level of visual impairment in patients with DED, which is consistent with findings using alternative methods.

\section{Functional Visual Acuity Measurement}

Functional visual acuity tests measure visual acuity during and after sustained visual activity. In this way, the tests are thought to be more representative of visual function in reallife situations such as driving, reading, and using a computer which require prolonged gazing [16, 17]. A purported advantage of FVA assessments is that they correlate with measures of optical surface irregularities, tear film breakup time, corneal opacity, and corneal vascularization [17, 18]. FVA tests appear to have some value in the assessment of visual impairment in patients with DED [16].

One FVA measure was first introduced in 2002, and showed a significant decrease in patients with non-Sjögren syndrome and Sjögren syndrome associated DED, despite 
no change in normal subjects [17]. The modification of the original FVA methods to make possible measurement from a greater distance and accommodate patients with lower baseline visual acuity scores demonstrated that patients with DED had significantly lower FVA compared with normal controls and the rate of decrease in FVA over time was faster in DED patients [19, 20]. In one such study, the reported average FVA of DED patients was 20/71 [17], which is just less than the acuity generally required for licensure for daytime driving in most US states (20/70), and worse than the widely accepted FVA cut point of 20/40 for unrestricted driving.

A study comparing patients with DED with normal subjects showed that FVA in DED was significantly lower than in control subjects at all time points $(10,20$, and $30 \mathrm{~s})$. This study also showed that FVA significantly improved after insertion of punctal plugs in DED patients at all time points [19]. A further study showed that not only the diagnosis of dry eye, but also the existence of superficial punctuate keratopathy resulted in deterioration of the visual function as measured by FVA [21]. The study showed a significant correlation between the severity of epithelial damage at the center of the cornea and variation of visual function, as well as coma-like and total higher-order aberrations (HOAs) [21].

\section{Measurement of Higher-Order Optical Aberrations}

There are two general classes of optical aberration: lowerorder aberration, which results from refractive errors, and HOA. A HOA is a distortion acquired by a wave front of light when it passes through an eye with irregularities of its refractive components such as the tear film, cornea, aqueous humor, crystalline lens, and vitreous humor. Optical aberration can be measured by both objective and subjective methods. Wave-front aberrometers assess real-time changes in the optics of the whole eye by measuring refractive anomalies at multiple locations using wave-front technology. Montes-Mico et al. [22] showed that, compared with normal controls, DED patients have greater optical aberrations due to increased tear film irregularity, which leads to an uneven corneal surface. Lin et al. [23] found that after laser-assisted in situ keratomileusis, eyes of DED patients have more rapid disruption of the tear film.

Koh et al. [24] found that the sequential postblink changes in the HOAs had a reverse sawtooth pattern when there was an excessive tear volume in a patient with dry eye who complained of paradoxical visual impairment with epiphora despite an improvement of the dry eye after punctal plug insertion. Denoyer et al. [25] proved that third-order aberrations, in particular, significantly increased over the 10-s period in DED patients, whereas no change occurred in controls. Analysis of the modulation transfer function revealed a progressive degradation of ocular optical quality resulting from loss of contrast sensitivity at intermediate and high spatial frequencies in DED patients.

\section{The Economic Impact of DED}

The burden of DED on the patient and society also includes a substantial economic burden, which can be divided into two categories: direct costs such as medical fees and indirect costs such as low employment, absence from work, and impaired productivity. Assessment of the economic burden of DED in the USA based on survey data collected from 2,171 respondents with DED showed that the average annual direct cost of treating a patient with DED is $\$ 783$ (range, \$757 to \$809), resulting in an overall annual burden of direct costs for DED for the US health care system of $\$ 3.84$ billion [54] Evaluating the cost-effectiveness of dry eye treatment is quite challenging owing to the multifactorial nature of the disease and potential limitations of techniques available to evaluate therapeutic outcomes of the multipalliative treatment modalities used. Treatment costs would be predicted to be affected by the availability of various treatments, the quality of the health care system, and the propensity of patients to seek and receive treatment, among other factors. Such predicted differences may be borne out in data such as those from a study that involved six European countries (France, Germany, Italy, Spain, Sweden, and the UK) showing that the total annual health care cost of treating 1,000 DED patients by ophthalmologists ranged from US $\$ 0.27$ million in France to $\$ 1.10$ million in the UK [55]. The cost included the cost of specialist visits, diagnostic tests, and pharmacological and nonpharmacological treatment. In Asia, Mizuno et al. [56] found in a multicenter study evaluating 118 dry eye patients that the annual drug cost was US\$323 \pm US $\$ 219$, the clinical cost was US\$165 \pm US\$101, and the total direct costs, including punctal plug treatment, amounted to US\$530 \pm US\$384. Investigators from the Singapore National Eye Centre evaluated the cost data from 54,052 patients and found that total annual expenditure on dry eye treatment for 2008 and 2009 exceeded US $\$ 1.5$ million, and the total expenditures per patient visit in 2008 and 2009 were US\$22.11 and US\$23.59 [57].

The indirects cost of DED due to work absenteeism (absence, early leaving) and presenteeism (equivalent lost work days because of affected performance, as proposed by Auren [58] in 1955 to describe productivity loss when employees come to work but are not fully productive) were estimated to average $\$ 11,302$ per year per patient with DED, with a corresponding overall US annual indirect cost burden of \$55.4 billion [54]. Absenteeism from work averaged 
8.4 days for patients with mild DED, to 14.2 days per year for patients with severe DED [54]. Presenteeism was equivalent to 91 days for patients with mild DED, 94.9 days for patients with moderate DED, and 128.2 days for patients with severe DED [54]. Reddy et al. [59] also reported that patients with dry eye take 2-5 days off work annually, whereas they were present at work having symptoms for 191-208 days annually, indicating that presenteeism is a greater issue than absenteeism among those having dry eye.

A study from Japan evaluated the impact of dry eye on work productivity of office workers, especially in terms of presenteeism, using a work-limitations questionnaire, an established tool for evaluation of presenteeism [60]. Among 396 individuals aged 20 years or older, the investigators showed that productivity in the group with self-reported DED was significantly lower than that in the control group $(P<0.05)$. The annual cost of work productivity loss associated with DED was estimated to be US\$741 per person [61]. Another cross-sectional, Web-based survey administered to 9,034 individuals aged 18 years or older using the OSDI and the Work Productivity and Activity Impairment Questionnaire [62] showed work productivity loss was related to the severity of DED. Patients with moderate DED $(18 \%)$ and severe DED (35\%) had significantly greater reductions in work productivity than patients with mild DED $(11 \%)$. Similarly, impairment in the ability to perform daily activities was significantly greater among respondents with severe DED (34 \%) than respondents with moderate DED $(19 \%)$ or mild DED (12\%) [63].

\section{Conclusion}

DED is a common and growing problem that results in a multifaceted degradation of patients' QoL and visual performance. In this review, we have summarized the literature describing the impact of DED on patients' QoL. Such studies consistently show that DED has a measurable impact on several aspects of patients' QoL, including pain, vitality, ability to perform certain activities requiring sustained visual attention (e.g., reading, driving), and productivity in the workplace. Research also shows a substantial economic impact of DED as a result of these QoL impacts. Treatment of DED should therefore aim to improve patients' QoL as this aspect of the disease appears to be the primary driver of its importance to both individual patients and society as a whole. It may be important to enhance awareness of dry eye among society by educational activities.

Disclosure M. Uchino: none; D.A. Schaumberg: has served as a board member for SARcode Bioscience, and Mimetogen, has served as a consultant for Eleven Biotherapeutics, Pfizer, Alcon, Allergan, Inspire Pharmaceuticals, and Resolvyx, has grants/grants pending from Pfizer, has received payment for development of educational presentations from Allergan, and has stock/stock options with Tear Lab and Mimetogen.

\section{References}

Papers of particular interest, published recently, have been highlighted as:

- Of importance

1. - The definition and classification of dry eye disease: report of the Definition and Classification Subcommittee of the International Dry Eye WorkShop (2007). Ocul Surf 2007;5:75-92. This review provides the fundamental and essential points to define and classify DED.

2. Bjerrum KB. Keratoconjunctivitis sicca and primary Sjogren's syndrome in a Danish population aged 30-60 years. Acta Ophthalmol Scand. 1997;75:281-6.

3. Schein OD, Munoz B, Tielsch JM, Bandeen-Roche K, West S. Prevalence of dry eye among the elderly. Am J Ophthalmol. 1997; 124:723-8.

4. McCarty CA, Bansal AK, Livingston PM, Stanislavsky YL, Taylor HR. The epidemiology of dry eye in Melbourne, Australia. Ophthalmology. 1998;105:1114-9.

5. Shimmura S, Shimazaki J, Tsubota K. Results of a populationbased questionnaire on the symptoms and lifestyles associated with dry eye. Cornea. 1999;18:408-11.

6. Moss SE, Klein R, Klein BE. Prevalence of and risk factors for dry eye syndrome. Arch Ophthalmol. 2000;118:1264-8.

7. Yazdani C, McLaughlin T, Smeeding JE, Walt J. Prevalence of treated dry eye disease in a managed care population. Clin Ther. 2001;23:1672-82.

8. - Schaumberg DA, Sullivan DA, Buring JE, Dana MR. Prevalence of dry eye syndrome among US women. Am J Ophthalmol 2003;136:318-26. This article estimates the prevalence DED among US women using data obtained from a cohort of more than 39,000 women.

9. Guo B, Lu P, Chen X, Zhang W, Chen R. Prevalence of dry eye disease in Mongolians at high altitude in China: the Henan eye study. Ophthalmic Epidemiol. 2010;17:234-41.

10. Schiffman RM, Christianson MD, Jacobsen G, Hirsch JD, Reis BL. Reliability and validity of the Ocular Surface Disease Index. Arch Ophthalmol. 2000;118:615-21.

11. Begley CG, Chalmers RL, Mitchell GL, et al. Characterization of ocular surface symptoms from optometric practices in North America. Cornea. 2001;20:610-8.

12. Rajagopalan K, Abetz L, Mertzanis P, et al. Comparing the discriminative validity of two generic and one disease-specific health-related quality of life measures in a sample of patients with dry eye. Value Health. 2005;8:168-74.

13. Huang FC, Tseng SH, Shih MH, Chen FK. Effect of artificial tears on corneal surface regularity, contrast sensitivity, and glare disability in dry eyes. Ophthalmology. 2002;109:1934-40.

14. Ridder WH 3rd, Tomlinson A, Paugh J. Effect of artificial tears on visual performance in subjects with dry eye. Optom Vis Sci. 2005;82:835-42.

15. Goto E, Ishida R, Kaido M, et al. Optical aberrations and visual disturbances associated with dry eye. Ocul Surf. 2006;4:207-13.

16. Kaido M, Dogru M, Ishida R, Tsubota K. Concept of functional visual acuity and its applications. Cornea. 2007;26:S29-35.

17. Goto E, Yagi Y, Matsumoto Y, Tsubota K. Impaired functional visual acuity of dry eye patients. Am J Ophthalmol. 2002;133:181-6.

18. Kaido M, Dogru M, Yamada M, et al. Functional visual acuity in Stevens-Johnson syndrome. Am J Ophthalmol. 2006;142:917-22. 
19. Kaido M, Ishida R, Dogru M, Tamaoki T, Tsubota K. Efficacy of punctum plug treatment in short break-up time dry eye. Optom Vis Sci. 2008;85:758-63.

20. Ishida R, Kojima T, Dogru M, et al. The application of a new continuous functional visual acuity measurement system in dry eye syndromes. Am J Ophthalmol. 2005;139:253-8.

21. Kaido M, Matsumoto Y, Shigeno Y, Ishida R, Dogru M, Tsubota $\mathrm{K}$. Corneal fluorescein staining correlates with visual function in dry eye patients. Invest Ophthalmol Vis Sci. 2011;52:9516-22.

22. Montes-Mico R, Caliz A, Alio JL. Wavefront analysis of higher order aberrations in dry eye patients. J Refract Surg. 2004;20: 243-7.

23. Lin YY, Carrel H, Wang IJ, Lin PJ, Hu FR. Effect of tear film break-up on higher order aberrations of the anterior cornea in normal, dry, and post-LASIK eyes. J Refract Surg. 2005;21: S525-9.

24. Koh S, Maeda N, Ninomiya S, et al. Paradoxical increase of visual impairment with punctal occlusion in a patient with mild dry eye. J Cataract Refract Surg. 2006;32:689-91.

25. Denoyer A, Rabut G, Baudouin C. Tear film aberration dynamics and vision-related quality of life in patients with dry eye disease. Ophthalmology. 2012;119:1811-8.

26. Begley CG, Caffery B, Chalmers RL, Mitchell GL. Use of the dry eye questionnaire to measure symptoms of ocular irritation in patients with aqueous tear deficient dry eye. Cornea. 2002;21: 664-70.

27. Li M, Gong L, Chapin WJ, Zhu M. Assessment of vision-related quality of life in dry eye patients. Invest Ophthalmol Vis Sci. 2012;53:5722-7.

28. Unlu C, Guney E, Akcay BI, Akcali G, Erdogan G, Bayramlar H. Comparison of ocular-surface disease index questionnaire, tearfilm break-up time, and Schirmer tests for the evaluation of the tearfilm in computer users with and without dry-eye symptomatology. Clin Ophthalmol. 2012;6:1303-6.

29. Portello JK, Rosenfield M, Bababekova Y, Estrada JM, Leon A. Computer-related visual symptoms in office workers. Ophthalmic Physiol Opt. 2012;32:375-82.

30. García-Catalán MR, Jerez-Olivera E, Benítez-Del-Castillo-Sánchez JM. Dry eye and quality of life. Arch Soc Esp Oftalmol. 2009;84:451-8.

31. Fairchild CJ, Chalmers RL, Begley CG. Clinically important difference in dry eye: change in IDEEL-symptom bother. Optom Vis Sci. 2008;85:699-707.

32. Begley CG, Chalmers RL, Abetz L, et al. The relationship between habitual patient-reported symptoms and clinical signs among patients with dry eye of varying severity. Invest Ophthalmol Vis Sci. 2003;44:4753-61.

33. Bjerrum KB. Test and symptoms in keratoconjunctivitis sicca and their correlation. Acta Ophthalmol Scand. 1996;74:436-41.

34. Vitali C, Moutsopoulos HM, Bombardieri S. The European Community Study Group on diagnostic criteria for Sjogren's syndrome. Sensitivity and specificity of tests for ocular and oral involvement in Sjogren's syndrome. Ann Rheum Dis. 1994;53: 637-47.

35. Toda I, Fujishima H, Tsubota K. Ocular fatigue is the major symptom of dry eye. Acta Ophthalmol (Cph). 1993;71:347-52.

36. Schaumberg DA, Dana R, Buring JE, Sullivan DA. Prevalence of dry eye disease among US men: estimates from the Physicians' Health Studies. Arch Ophthalmol. 2009;127:763-8.

37. - Miljanovic B, Dana R, Sullivan DA, Schaumberg DA. Impact of dry eye syndrome on vision-related quality of life. Am J Ophthalmol 2007;143:409-415. This article was among the first to demonstrate the impact of DED on vision-related $Q o L$.

38. Tong L, Waduthantri S, Wong TY, et al. Impact of symptomatic dry eye on vision-related daily activities: the Singapore Malay Eye Study. Eye (Lond). 2010;24:1486-91.
39. Nease RF, Whitcup SM, Ellwein LB, Fox G, Littenberg B. Utility-based estimates of the relative morbidity of visual impairment and angina. Ophthalmic Epidemiol. 2000;7:169-85.

40. Buchholz P, Steeds CS, Stern LS, et al. Utility assessment to measure the impact of dry eye disease. Ocul Surf. 2006;4:155-61.

41. Schiffman RM, Walt JG, Jacobsen G, Doyle JJ, Lebovics G, Sumner W. Utility assessment among patients with dry eye disease. Ophthalmology. 2003;110:1412-9.

42. McHorney CA, Ware JE Jr, Raczek AE. The MOS 36-item shortform health survey (SF-36): II. Psychometric and clinical tests of validity in measuring physical and mental health constructs. Med Care. 1993;31:247-63.

43. Mertzanis P, Abetz L, Rajagopalan K, et al. The relative burden of dry eye in patients' lives: comparisons to a U.S. normative sample. Invest Ophthalmol Vis Sci. 2005;46:46-50.

44. Moss SE, Klein R, Klein BE. Long-term incidence of dry eye in an older population. Optom Vis Sci. 2008;85:668-74.

45. Schein OD, Hochberg MC, Munoz B, et al. Dry eye and dry mouth in the elderly: a population-based assessment. Arch Intern Med. 1999;159:1359-63.

46. Mangione CM, Lee PP, Pitts J, Gutierrez P, Berry S, Hays RD. Psychometric properties of the National Eye Institute Visual Function Questionnaire (NEI-VFQ). NEI-VFQ field test investigators. Arch Ophthalmol. 1998;116:1496-504.

47. Le Q, Zhou X, Ge L, Wu L, Hong J, Xu J. Impact of dry eye syndrome on vision-related quality of life in a non-clinic-based general population. BMC Ophthalmol. 2012;12:22.

48. Nichols KK, Mitchell GL, Zadnik K. Performance and repeatability of the NEI-VFQ-25 in patients with dry eye. Cornea. 2002;21:578-83.

49. Vitale S, Goodman LA, Reed GF, Smith JA. Comparison of the NEI-VFQ and OSDI questionnaires in patients with Sjögren's syndrome-related dry eye. Health Qual Life Outcomes. 2004;2:44.

50. Rossi GC, Tinelli C, Pasinetti GM, Milano G, Bianchi PE. Dry eye syndrome-related quality of life in glaucoma patients. Eur J Ophthalmol. 2009;19:572-9.

51. Leung EW, Medeiros FA, Weinreb RN. Prevalence of ocular surface disease in glaucoma patients. J Glaucoma. 2008;17:350-5.

52. Rolando M, Iester M, Macri A, Calabria G. Low spatial-contrast sensitivity in dry eyes. Cornea. 1998;17:376-9.

53. Ridder WH 3rd, Tomlinson A, Huang JF, Li J. Impaired visual performance in patients with dry eye. Ocul Surf. 2011;9:42-55.

54. Yu J, Asche CV, Fairchild CJ. The economic burden of dry eye disease in the United States: a decision tree analysis. Cornea. 2011;30:379-87.

55. Clegg JP, Guest JF, Lehman A, Smith AF. The annual cost of dry eye syndrome in France, Germany, Italy, Spain, Sweden and the United Kingdom among patients managed by ophthalmologists. Ophthalmic Epidemiol. 2006;13:263-74.

56. Mizuno Y, Yamada M, Shigeyasu C. Annual direct cost of dry eye in Japan. Clin Ophthalmol. 2012;6:755-60.

57. Waduthantri S, Yong SS, Tan $\mathrm{CH}$, et al. Cost of dry eye treatment in an Asian clinic setting. PLoS ONE. 2012;7:e37711.

58. Auren U. How to build presenteeism. Pet Refiner. 1955;34:12.

59. Reddy P, Grad O, Rajagopalan K. The economic burden of dry eye: a conceptual framework and preliminary assessment. Cornea. 2004;23:751-61.

60. Lerner D, Amick BC 3rd, Rogers WH, Malspeis S, Bungay K, Cynn D. The work limitations questionnaire. Med Care. 2001;39:72-85.

61. Yamada M, Mizuno Y, Shigeyasu C. Impact of dry eye on work productivity. Clinicoecon Outcomes Res. 2012;4:307-12.

62. Reilly MC, Zbrozek AS, Dukes EM. The validity and reproducibility of a work productivity and activity impairment instrument. Pharmacoeconomics. 1993;4:353-65.

63. Patel VD, Watanabe JH, Strauss JA, Dubey AT. Work productivity loss in patients with dry eye disease: an online survey. Curr Med Res Opin. 2011;27:1041-8. 